\title{
As informações de pagamento influenciam meu parcelamento? Uma análise do efeito da divulgação das informações das condições de compra a crédito na propensão do consumo a prazo
}

Does the information disclosure matters in my credit purchase?: An analysis of the effect of information credit disclore in the credit propensity of purchase

Frederike Monika Budiner Mette ${ }^{1}$

\section{Resumo}

Este artigo teve como objetivo geral analisar qual o efeito causado pela divulgação das informações das condições de compra a crédito de um determinado produto, por meio de dois níveis, na propensão de os indivíduos consumirem este crédito. Além disso, busca-se analisar se essa relação é moderada pelos fatores "conhecimento financeiro sobre o crédito" (debt literacy) e "poder de compra para pagamento à vista". Como procedimento metodológico, realizou-se um experimento com a manipulação da variável "divulgação das informações das condições de compra a crédito", com uma amostra total de 60 pessoas. Dentre as principais conclusões, verificou-se que uma maior quantidade de informações das condições de compra a crédito de um determinado produto resulta em uma maior propensão de os indivíduos consumirem esse crédito, efeito inverso ao esperado. Assim como em um cenário com maior quantidade de informações das condições de compra a crédito, o nível de conhecimento financeiro de dívidas tende a aumentar a propensão de os indivíduos consumirem a prazo.

Palavras-chave: Divulgação das informações. Compra a crédito. Propensão de compra. Experimento

Graduada em Ciências Atuariais pela UFRGS, mestre em ADM com ênfase em Finanças pelo PPGA/UFRGS e doutoranda em ADM com ênfase em Marketing pelo PPGA/UNISINOS, com doutorado-sanduíche pela University of Oxford - Brasil - E-mail: frederikemette@yahoo.com.br 


\section{Abstract}

This article aimed to analyze the effect that is caused by the disclosure of credit terms in buying a particular product, measured in two levels the propensity of individuals to consume some credit. In addition, it has analyzed whether this relationship is moderated by debt literacy and purchasing power in cash. It was an experiment that manipulated the information of credit buying conditions ina sample of 60 people. The main findings were that a greater amount of information in the credit purchase conditions results in a greater propensity for individuals to consume this credit, meaning the expected inverse effect. And in a scenario with much information on credit buying conditions, the level of financial debt knowledge tends to increase the propensity of individuals to consume on credit.

Keywords: Information disclosure. Credit purchase. Propensity of purchase. Experiment

\section{Introdução}

No Brasil, na última década, ocorreu um elevado crescimento no nível de endividamento da população, com o volume de crédito atingindo $54,1 \%$ do PIB brasileiro em 2013. Outro fato relevante é que a inadimplência das pessoas físicas alcançou 19,5\% em janeiro de 2014 (BACEN, 2014). Já o nível de famílias brasileiras endividadas alcançou $61 \%$ em março de 2014 . O cartão de crédito foi apontado como um dos principais tipos de dívida por $74,2 \%$ das famílias endividadas, seguido por carnês $(17,5 \%)$, e, em terceiro, financiamento de carro $(14,0 \%)$ (PEIC, 2014). Além disso, a partir da crise de 2008, a redução do ritmo de crescimento da economia, a aceleração inflacionária e a queda da renda real da população contribuíram para a piora dos indicadores de endividamento e inadimplência.

Assim, as decisões de tomada de consumo a crédito, ou seja, de fatores de endividamento por parte dos indivíduos brasileiros, têm sido um dos assuntos mais pesquisados e comentados ultimamente (PEIC, 2014). Face aos atuais dados sobre o mercado de crédito, endividamento e inadimplência no mercado brasileiro, tornam-se importantes pesquisas que busquem entender o comportamento do consumidor em decisões de compras a prazo e os fatores que as influenciam. 
Nesse contexto, objetivando identificar o efeito de mensagens publicitárias no processo de tomada de decisão dos indivíduos, Tonetto (2009) identificou que o grau de persuasão dos indivíduos expostos a textos salientando as vantagens do uso de cartão de crédito frente ao dinheiro e ao cheque tende a ser superior ao grau de persuasão de um material que exalte as desvantagens do uso de dinheiro e cheque em relação aos cartões de crédito. O autor afirma que, apesar de o conteúdo dos dois materiais ser equivalente, a mensagem gera diferentes níveis de persuasão nas pessoas, por serem configuradas de formas distintas.

Como alternativa para um consumo mais consciente, Bolton, Cohen e Bloom (2011) afirmam que uma intervenção no nível de educação financeira, em que são dadas informações sobre os empréstimos e financeiras, pode ajudar os consumidores a entenderem e responderem de melhor maneira à consolidação das mídias de consolidação de crédito, pois dessa forma reduziria o risco moral nas negociações, em que a financeira "vende" a ideia de que postergar as dívidas seria o remédio para a saúde financeira dos indivíduos.

Conjuntamente, Souza (2013) afirma que, embora haja um esforço claro para educar financeiramente a população, pouca ênfase tem sido dada aos fatores preditivos e consequentes do uso de crédito em termos cognitivos, atitudinais e motivacionais dos próprios consumidores. $\mathrm{Ou}$ seja, busca-se uma consolidação da educação financeira como solução de um endividamento, entretanto, precisa-se estudar também os outros fatores que afetam a intenção de compra a crédito.

Portanto, com o objetivo de suprir algumas das lacunas existentes na pesquisa sobre os fatores preditores do endividamento da população, este estudo questiona: qual o efeito da divulgação das condições de compra a crédito de um determinado produto na propensão de o indivíduo consumir este a crédito? Nessa relação, existe alguma moderação de variáveis como debt literacy e a possibilidade de pagamento à vista?

Assim, o estudo pretende verificar qual o efeito causado pela divulgação das informações das condições de compra a crédito de um determinado produto, por meio de dois níveis, na propensão de os 
indivíduos consumirem este crédito. Além disso, busca-se analisar se essa relação é modelada pelos fatores "conhecimento financeiro sobre o crédito" (debt literacy) e "poder de compra para pagamento à vista".

A seguir, apresenta-se o referencial teórico referente ao processo de tomada de decisão de consumo, o efeito das mensagens, a sustentação e a apresentação das hipóteses propostas neste estudo. Depois, é apresentado o método empregado na pesquisa e, finalmente, são relatados os resultados e as considerações finais desta pesquisa.

\section{O efeito da divulgação das informações das condições de compra a crédito no processo de tomada de decisão de compra}

O efeito framing, ou efeito de configuração, se refere às concepções das pessoas frente às tarefas decisórias, aos atos, resultados e às contingências associadas com suas escolhas (TVERSKY \& KAHNEMAN, 1981). Assim, o efeito de configuração refere-se ao entendimento de que as pessoas respondem de formas distintas a diferentes caracterizações de um mesmo problema decisório (TONETTO et al., 2010).

Para Tonetto et al. (2010), o efeito de configuração de objetivos envolve o grau de persuasão de um conteúdo comunicado para ações ou comportamentos. Nessa direção, o impacto de uma comunicação persuasiva tem se mostrado dependente do fato de a mensagem enfatizar consequências positivas ao realizar um comportamento ou consequências negativas ao não realizá-lo. No cenário atual, os autores afirmam que o correto seria falar em efeitos de configuração, considerando as distintas formas que o fenômeno tomou: configuração de risco, de objetivos e de atributos.

Partindo da definição de Kamleitner, Hoelzl e Kirchler (2012), o processo de aquisição de produtos inicia-se na fase que antecede a decisão da compra, sendo esta altamente influenciada pelos aspectos psicológicos. Por isso, é necessário identificar como o consumidor coleta e interpreta as informações do crédito para a sua decisão de consumo. 
Aliás, esses dados tornam-se essenciais quando se busca identificar o efeito que as promoções incentivadoras do consumo a crédito têm sobre essas decisões.

Ou seja, conforme as definições apresentadas por Tversky e Kahneman (1981), uma compra a crédito poderia ser uma manifestação de framing (efeito de configuração), porque o indivíduo dá preferência à aquisição de um produto com pagamento postergado devido à sensação de posse do dinheiro sem a associação a futuros pagamentos e taxas de juros. Dessa forma, considerando os estímulos existentes em anúncios publicitários para compra de produtos e oferecimento de crédito, é possível verificar que o indivíduo é constantemente avivado para a compra, verificando a persuasão que a mensagem pode ter na propensão dos consumidores utilizarem um crédito oferecido.

Nos estudos sobre quantidade de informações, as descrições expandidas de problemas decisórios são entendidas como aquelas que contêm os argumentos completos em relação às alternativas em escolha, enquanto que as resumidas são consideradas versões não completas dos mesmos problemas (KÜHBERGER, 1995). Portanto, presume-se que, à medida que se tem informações completas sobre uma determinada operação a crédito, a decisão por parte dos indivíduos tende a não ser meramente intuitiva, isto é, eles devem utilizar um caminho mais lento e racional. Afinal, Kahneman (2002) já enfatizava que as pessoas realizam um erro de estimativa devido à maneira como acreditam dar conta de suas obrigações futuras. Ocorrendo uma falta de programação para liquidar as parcelas com vencimento futuro, conhecida como fenômeno de falácia do planejamento, definido por Kahneman e Tversky (1979), esse problema foi apresentado como uma heurística ou viés que influenciam o processo de tomada de decisão financeira individual.

Analisando o efeito da divulgação de condições de compra a crédito, Bolton, Cohen e Bloom (2006) concluíram em sua pesquisa que a exposição dos indivíduos a uma propaganda incentivadora de consolidação das dívidas reduz o risco financeiro percebido para uma 
operação, aumentando as intenções do comportamento de compra a prazo, ou seja, de consumo de produtos de forma parcelada ou a crédito. Portanto, percebe-se que, de acordo com o formato de divulgação de algum crédito, as pessoas tendem a consumir mais ou não, levandoas a interpretar essas informações de modo heurístico, pois aquilo que incentiva o consumo é a propaganda, e não as características do crédito em si.

Adicionalmente, Bloom, Bolton e Cohen (2010) encontraram poucas propagandas e publicidades de empréstimos que mencionassem as altas taxas de juros e os longos prazos; pelo contrário, a maioria enfatizava que os pagamentos mensais seriam baixos, fato comumente visualizado no Brasil.

Assim sendo, pode-se afirmar que o modo da divulgação das condições de compra a crédito de um produto é uma configuração de objetivos, em que se verifica a comparação das avaliações dos sujeitos entre os resultados de realizar o comportamento ou não realizá-lo. Nesse caso, será medido o comportamento relacionado à intenção de compra a prazo.

H1: Quanto maior a quantidade de informações sobre as condições de compra a crédito, menor a propensão de o indivíduo consumir a crédito.

Essa mesma relação causal pode ser analisada por meio da moderação de diferentes fatores que podem afetar esse efeito principal. Williams, Fitzsimons e Block (2004) afirmaram que, quando os consumidores são educados financeiramente, percebem que um questionamento proposital em um anúncio pode ser uma tentativa de persuasão e que este comportamento de consumo seria atenuado. Portanto, é possível haver um ajuste de comportamento à medida que ocorre uma assimilação do objetivo da informação dada.

Nesse sentido, Lyons (2007) afirma que os estudantes com pouca experiência financeira ou com conhecimentos financeiros limitados não entenderiam conceitos financeiros básicos, como o efeito cumulativo da 
taxa de juros sobre a dívida com cartão de crédito, aumentando seu risco de problemas financeiros. Assim, de acordo com Beal e Delpachitra (2003), as consequências de um baixo nível de conhecimento financeiro são o uso exagerado de cartão de crédito (comportamento irresponsável) e a maior propensão a um mau gerenciamento do cartão (endividamento).

Portanto, espera-se que, à medida que o conhecimento financeiro sobre dívidas - debt literacy - entre os indivíduos seja alto, o acúmulo de conhecimento adquirido por meio da educação e/ou experiência relacionada a conceitos e produtos financeiros influencie no momento da decisão de comprar a prazo. Ou seja, se houver um maior detalhamento das condições de realizar esse ato, o indivíduo demorará mais para decidir pelo parcelamento; assim, sua intenção de compra a prazo deve ser menor. Portanto, presume-se que o nível de conhecimento financeiro tem um efeito negativo sobre uma intenção de compra a prazo.

H2: Quanto maior o nível de conhecimento financeiro de dívidas (debt literacy),

H2a: em um cenário com informações completas sobre a compra a crédito, muito menor a propensão de compra a crédito;

H2b: em um cenário real das informações sobre a compra a crédito, menor a propensão de compra a crédito.

Além disso, considera-se que, se um indivíduo tiver condições de pagar à vista pelo produto que está comprando, ele não terá interesse em se endividar, pois estará sem um compromisso financeiro futuro e não estará exposto a qualquer risco de pagamentos de juros e multa, em situações de atraso.

H3: Quanto maior a possibilidade de pagamento à vista, menor a propensão de compra a crédito, independentemente da quantidade de informações sobre a compra a prazo.

A partir das três hipóteses construídas, propõe-se o seguinte modelo teórico para o experimento a ser realizado esta pesquisa, conforme demonstrado na Figura 1. 
Figura 1 - Modelo teórico do experimento

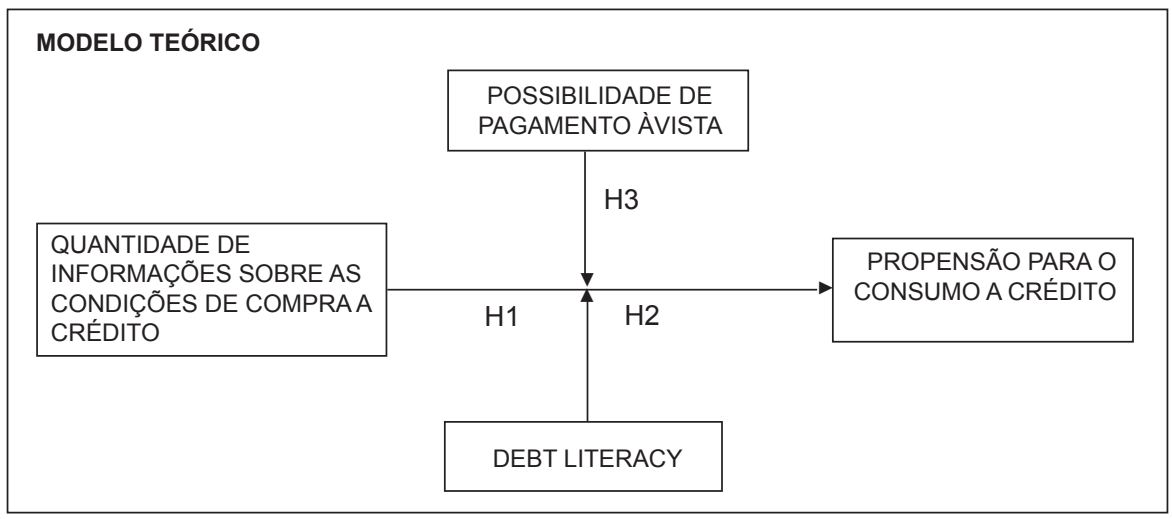

Fonte: Elaborado pela autora.

A seguir, tem-se o objetivo de apresentar um detalhamento do método e do experimento realizado, a fim de testar as hipóteses criadas.

\section{Método}

As hipóteses apresentadas na seção anterior serão investigadas por meio de um experimento que tem como objetivo analisar o efeito da divulgação das condições de compra a crédito de um determinado produto na propensão de o indivíduo consumir este a crédito.

Desenho e participantes: este experimento foi estruturado na forma de um desenho single-factor, between-subjects. Responderam ao estudo, com distribuição aleatória dos cenários aos respondentes, 36 indivíduos para o grupo de tratamento - Grupo 1 e 24 para o grupo de controle - Grupo 2, totalizando 60 pessoas na amostra já validada.

Procedimento e estímulo: as pessoas foram convidadas a responder ao questionário através da internet, havendo alocação dos cenários de forma aleatória por meio do software Qualtrics ${ }^{\circledR}$. Criaram-se três níveis de manipulação para a variável independente (divulgação das informações sobre as condições de compra a crédito em três configurações), conforme descrito na Figura 2. O grupo de 
tratamento recebeu a "informação completa" e o grupo de controle, os "requerimentos mínimos", pelo mínimo legal exigido, como definido pela lei 5.903/2006. Os grupos de controle seguem o layout de uma propaganda real, mantendo a validade externa do cenário. Para o Grupo 1 , informa-se todos os detalhes referentes à operação a prazo, como informações da financeira, taxas de juros e multas em caso de atraso e cláusulas do contrato. Além da manipulação dos cenários como variáveis independentes, tem-se a variável dependente (propensão de consumo a crédito) e dois moderadores (debt literacy e possibilidade de pagamento à vista).

Figura 2 - Manipulação da variável independente

\begin{tabular}{|l|l|l|}
\hline Informação financeira em propaganda & $\begin{array}{c}\text { Informação Completa } \\
\text {-GRUPO DE } \\
\text { TRATAMENTO (1) }\end{array}$ & $\begin{array}{c}\text { Requerimentos } \\
\text { Mínimos - } \\
\text { GRUPOS DE } \\
\text { CONTROLE (2) }\end{array}$ \\
\hline Quantidade de parcelas & & \\
\hline Valor das parcelas & & \\
\hline Taxa de juros & & \\
\hline Multa em caso de pagamento mínimo & & \\
\hline Informações sobre a financeira responsável & & \\
\hline Dados de contato & & \\
\hline Cláusulas de atraso de pagamento & & \\
\hline Valor à vista quando aplicado um desconto para o mesmo & & \\
\hline
\end{tabular}

Fonte: Elaborado pela autora.

Medidas: os participantes indicaram sua propensão de compra a crédito do produto por meio de uma escala de intenção de compra, com 3 itens e 7 escalas, criada por Burton, Garretson e Velliquette (1999). A medida para checar a manipulação dos cenários, pelo nível de divulgação de informação, foi por uma escala de 1 para pouca informação até 7 pontos para muita informação ("Em sua opinião, qual o nível de informação sobre o parcelamento de compra, que aparecia no anúncio anterior?"). Para a medição dos moderadores, utilizou-se: a) no constructo que mensura a possibilidade para pagamento à vista, solicitouse ao respondente que respondesse "Eu teria dinheiro disponível para efetuar o pagamento à vista, caso quisesse mesmo comprar uma das TVs que aparecem no anúncio", com uma escala de 1 para discordo 
e 7 para concordo; b) o constructo debt literacy utilizou um indicador criado por Lusardi e Tufano (2009), validado no Brasil por Souza (2013) e adaptado para esta pesquisa, que indica a habilidade do consumidor de responder corretamente a questionamentos sobre taxas de juros, dívidas de pagamento do cartão de crédito, pagamentos mínimos do cartão e valor do dinheiro no tempo. Como mensuração para o nível de conhecimento financeiro, haverá um número máximo de acertos 3 (alto conhecimento), 2 (conhecimento intermediário), 1 (baixo conhecimento) e 0 (nenhum).

\section{Análise dos resultados}

Para a análise dos resultados, primeiramente verificou-se a propensão ao consumo de crédito por meio do efeito principal, para, posteriormente, ser realizada a análise do efeito dos moderadores propostos neste estudo.

A análise de variância (ANOVA) foi realizada para mensurar o efeito da relação entre a quantidade de informações sobre a compra a prazo de televisores e a propensão de consumo a crédito $(\mathrm{H} 1)$, sendo esta significativa $(F(1,58)=6,2924 ; p<0,01)$. Com relação ao Grupo 1 (divulgação das informações completa), quando os respondentes estavam expostos a todas as condições da compra a prazo, eles apresentaram uma maior propensão de compra a crédito ( $\left.M_{\text {Grupo } 1}=4,12\right)$; no momento em que o grupo submetido aos requerimentos mínimos legais, conforme exibido nos meios de divulgação de lojas, tiveram uma propensão de compra a crédito menor $\left(\mathrm{M}_{\text {Grupo }} 2=2,89\right)$ (Figura 33).

Assim, conclui-se, segundo os resultados da pesquisa, que quanto maior a quantidade de informações sobre as condições de compra a crédito, maior a propensão de o indivíduo consumir a crédito. Esses dados refutam a $\mathrm{H} 1$ e mostram um efeito inverso ao esperado com significância. 
Figura 3 - Relação entre os grupos e a propensão de consumo a crédito

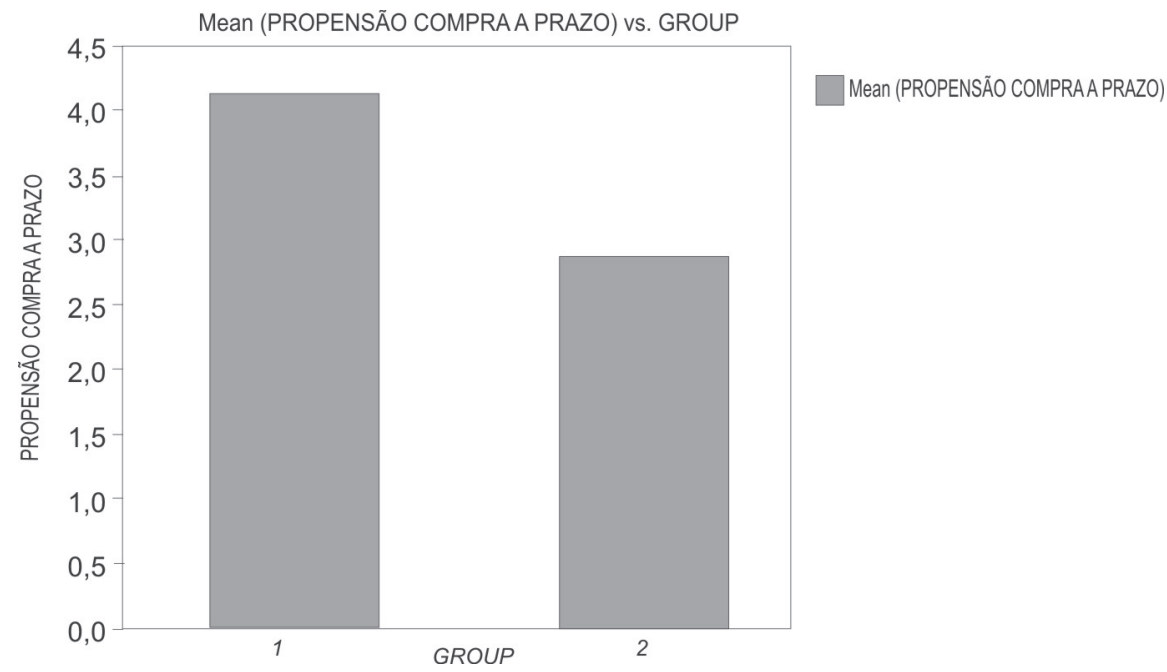

Fonte: Dados da pesquisa.

Para a análise dos moderadores, por serem variáveis contínuas, utilizou-se como ferramenta de análise o Spotlight, por meio de uma simulação de dados pelo Boostrap, a um intervalo de 95\% de confiança.

Aanálise para verificar se relação entre a quantidade de informações sobre as condições de compra a crédito e a propensão de consumo a crédito é moderada pela variável debt literacy se mostrou significativa com $(F(3,56)=3,34, p<0,05)$, havendo um aumento no nosso efeito com a interação entre a variável independente e o moderador. A interação se mostra significante a partir de nível de conhecimento financeiro de dívidas no valor de 1,06, isto é, o moderador influencia a propensão de compra a crédito para pessoas que possuem um nível de debt literacy.

Analisando as médias, percebe-se, por exemplo, que com um nível de conhecimento financeiro de dívidas na escala de 1,05, tem-se uma média de propensão a consumo a crédito: no Grupo 1, de 4,10; no Grupo 2, de 3,17. E na maior escala, de 2,5 pontos, tal média é de 4,32 para o Grupo 1 e 2,42 para o Grupo 2 (Figura 3). 
Figura 4 - Relação entre os grupos e a propensão de consumo a crédito para a moderação de debt literacy

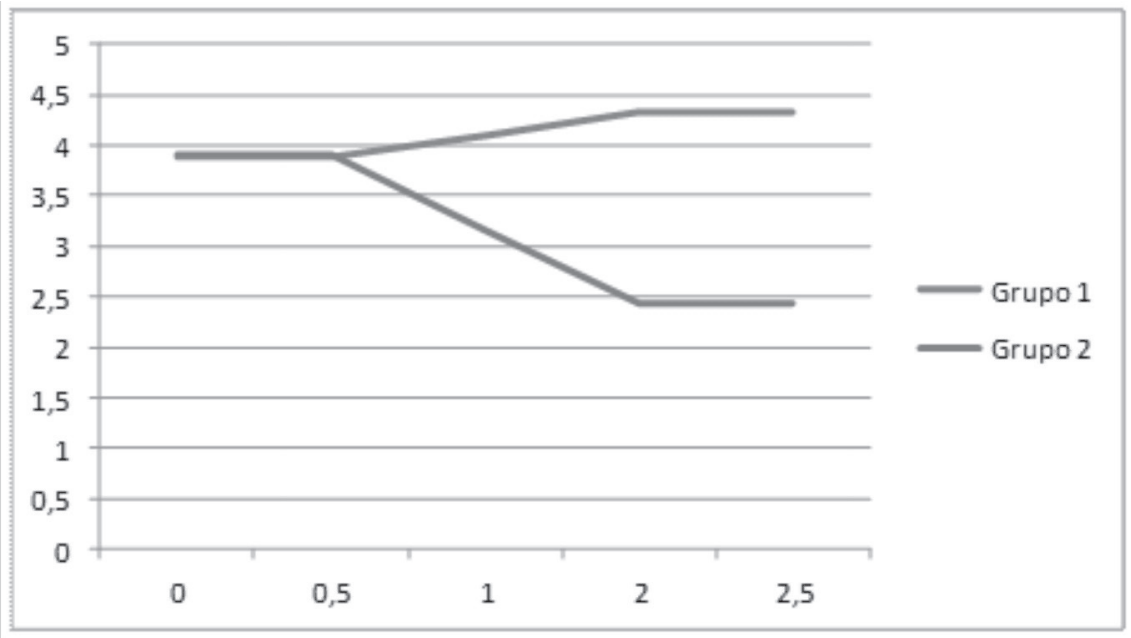

Fonte: Elaborado pelo autor.

Dessa forma, percebe-se que quando o indivíduo recebe uma informação completa sobre as condições de compra a prazo (Grupo 1), um maior conhecimento financeiro sobre dívidas tende a aumentar sua propensão de compra a prazo. Para um cenário real da demonstração das condições de compra a prazo (Grupo 2), um maior conhecimento financeiro sobre dívidas tende a reduzir sua propensão de compra a prazo. Assim, aceita-se $\mathrm{H} 2 \mathrm{~b}$; e para a $\mathrm{H} 2 \mathrm{a}$, temos o efeito inverso daquele previsto, de forma significativa.

A análise para verificar se relação entre a quantidade de informações sobre as condições de compra a crédito e a propensão de consumo a crédito é moderada pela possibilidade de pagamento à vista se mostrou significativa com $(F(3,56)=5,95, p<0,05)$, havendo um aumento do $\mathrm{R} 2$ do modelo com a interação entre a variável independente e o moderador. A interação se mostra significante a partir de uma possibilidade de pagamento à vista no valor de 5,2 , isto é, o moderador influencia a propensão de compra a crédito para pessoas que teriam possibilidade de pagamento à vista do televisor. 
Analisando as médias, percebe-se, por exemplo, que com uma possibilidade de pagamento à vista na escala de 5,2, tem-se uma média de propensão a consumo a crédito: no Grupo 1, de 3,94; no Grupo 2, de 3,12 . E na maior escala (7 pontos), tal média é de 3,62 para o Grupo 1 e 1,89 para o Grupo 2 (Figura 5).

Figura 5 - Relação entre os grupos e a propensão de consumo a crédito para a moderação de possibilidade de pagamento à vista

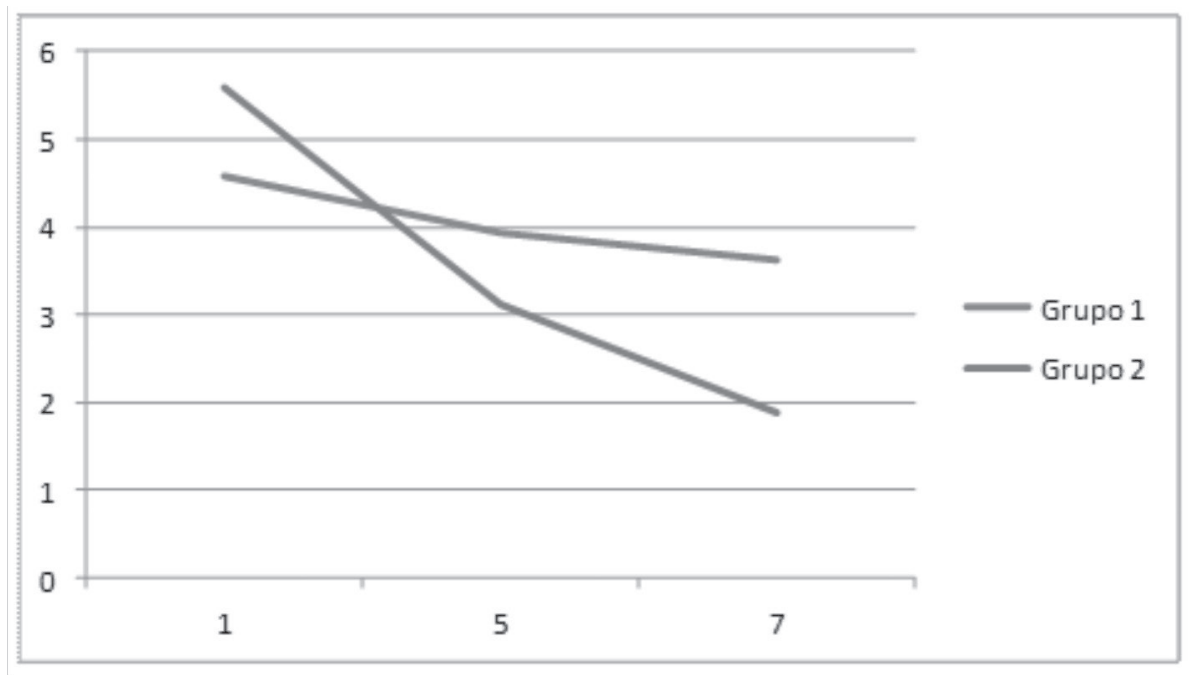

Fonte: Elaborado pelo autor.

Quando o indivíduo recebe uma informação completa sobre as condições de compra a prazo (Grupo 1), uma maior propensão de pagamento à vista tende a reduzir sua propensão de compra a prazo. Para um cenário real da demonstração das condições de compra a prazo (Grupo 2), uma maior propensão de pagamento à vista também tende a reduzir sua propensão de compra a prazo, porém, em uma proporção menor do que para o Grupo 1. Portanto, a hipótese H3 é aceita. 


\section{Considerações finais}

Realizou-se um experimento com a manipulação de dois cenários para a divulgação das informações de compra a crédito, em que se apresentou, de forma aleatória, o Grupo 1 (com informações completas sobre o crédito) e o Grupo 2 (de controle, com os requerimentos mínimos, por meio de uma propaganda real). A amostra foi aleatória e, após validada, resultou em 36 pessoas para o Grupo 1 e 24 pessoas no Grupo 2, totalizando 60 pessoas.

A partir dos resultados apresentados, para a hipótese $\mathrm{H} 1$, houve uma comprovação do efeito entre a variável independente sobre a dependente, porém, com um efeito reverso ao esperado, pois se identificou que os indivíduos que visualizavam uma maior quantidade de informações sobre as condições de compra a crédito apresentavam uma maior a propensão de consumir este crédito. Provavelmente, uma das razões pelas quais se obteve tal resultado foi a confiança criada com a instituição financeira quando se expõe as pessoas a uma total divulgação das informações. Isto é, em um cenário real (Grupo 2), o consumidor pode ficar menos confiante a adquirir a crédito o produto.

Pode-se concluir que, mesmo em um cenário com informações mais completas sobre a operação a crédito e a especificação dos riscos dessa operação, o indivíduo pode manter sua decisão de forma intuitiva, isto é, ele continua algum processo automático e deliberado para esse consumo.

Para o teste da $\mathrm{H} 2$, percebeu-se que quando o indivíduo recebe uma informação completa sobre as condições de compra a prazo (Grupo 1), um maior conhecimento financeiro sobre dívidas tende a aumentar sua propensão de compra a prazo. Para um cenário real da demonstração das condições de compra a prazo (Grupo 2), um maior conhecimento financeiro sobre dívidas tende a reduzir sua propensão de compra a prazo. Novamente, para a $\mathrm{H} 2 \mathrm{a}$, tem-se o efeito inverso daquele previsto, de forma significativa. 
Ou seja, grande parte da literatura afirma que quanto maior o volume de informações sobre as operações e mais educado financeiramente forem os indivíduos, mais racionais tendem a ser suas decisões de consumo envolvendo taxa de juros, por exemplo, pois nesse caso eles seriam um pouco mais avessos aos riscos exógenos que envolvem essas operações. Nesta pesquisa, identificou-se que, mesmo com um nível completo de informações sobre o risco financeiro das operações a crédito, o sujeito tende a aumentar sua intenção de compra a prazo à medida que tiver mais conhecimento financeiro sobre dívidas.

Parte desses resultados pode ser justificada pela confiança, já descrita, e uma maior autoconfiança que esse conhecimento financeiro pode gerar nessas pessoas, por meio de um maior autocontrole.

Finalmente, para $\mathrm{H} 3$, tem-se os resultados significativos no Grupo 1 e Grupo 2. Dessa forma, pode-se afirmar que quanto maior a possibilidade de pagamento à vista, menor a propensão de consumo a crédito, aceitando a hipótese inicialmente proposta. Assim, se um indivíduo possui possibilidade de pagamento à vista, independentemente do cenário que está exposto, sua intenção de compra a prazo será menor.

Esses resultados contribuem para a literatura de marketing e psicologia social, principalmente no que se refere à necessidade de mais estudos voltados a entender os comportamentos de consumos a crédito dos indivíduos, seus endividamentos e superendividamentos. Ou seja, como se pode estimar quais são as variáveis que interferem no processo de tomada de decisão a crédito?

Pelo que se percebe no estudo, é difícil construir um padrão de comportamento baseando-se somente em variáveis mais racionais, como possibilidade de pagamento à vista, nível de conhecimento financeiro de dívidas (debt literacy) e quantidade de informações na divulgação de compras a crédito. Isto é, presume-se que há outras variáveis que influenciam tal comportamento e não foram mensuradas no estudo, como comportamentais e de atitude. 
Do ponto de vista gerencial, este estudo vem contribuir com parte das atribuições emergentes quando o assunto é endividamento populacional. Ou seja, percebe-se que há diversas frentes para as empresas e governos atuarem com o objetivo de prevenir um superendividamento populacional, pois não basta somente educar os indivíduos se suas atitudes não se baseiam unicamente em seus conhecimentos.

Entretanto, é possível apresentar algumas limitações do estudo, como a criação e aplicação da questão de checagem, que comprovou não ser totalmente eficiente. Sugere-se utilizar uma medida de alfabetização financeira da população, em vez de conhecimento financeiro de dívidas, manipulando esses cenários com diferentes produtos, diferenciando-os por meio do envolvimento entre os sujeitos e estes, e aplicando-os de forma presencial. Porém, tal estudo vem para ratificar a importância de novos estudos que tentem desvendar o comportamento de consumo de crédito e o endividamento individual.

\section{Referências}

Banco Central do Brasil. Disponível em: <http://www.bcb.gov. br/?COPOMJUROS>. Acessado em: 22 de maio de 2014.

Beal, D. J.; Delpachitra, S. B. (2003) Financial literacy among Australian university students. Economic Papers, v. 22, n. 1, p. 65-78.

Bloom, Paul N., Bolton, Lisa E. e Cohen, Joel B. (2010). Helping Consumers Respond Responsibly to the Advertising and Availability of Debt Consolidation Loans. Interim Reports to the National Endowment for Financial Education.

Bolton, Lisa E., Cohen, Joel B., e Bloom, P. N. (2006). Does marketing products as remedies create "get out of jail free cards?. The Journal of Consumer Research, 33(1), 71-81.

Burton, Scot, Judith A. Garretson, and Anne M. Velliquette (1999), "Implications of Accurate Usage of Nutrition Facts Panel Information 
for Food Product Evaluations and Purchase Intentions," Journal of the Academy of Marketing Science, 27 (Fall), 470-80.

Kamleitner, B.; Hoelzl, E., e Kirchler, E. (2012): Credit use: Psychological perspectives on a multifaceted phenomenon. International Journal of Psychology, 47(1), 1-27.

Kahneman, D. (2002). Maps of Bouded Rationality: A Perspective on Intuitive Judgment and Choice. Local: editora???

Kahneman, D. e Tversky, A. (1979). Intuitive prediction: Biases and corrective procedures. Management Science, 12, 313-327.

Kühberger, A. (1995). The framing of decisions: a new look for old problems. Organizational Behavior and Human Decision Processes, 62(2), 230-240.

Lusardi, Annamaria e Tufano, Peter (2009). Debt Literacy, Financial Experiences, and Overindebtedness. NBER Working Paper $n$. 14808.

Lyons, A. C. (2007) Credit practices and financial education needs of Midwest college students. In: Social Science Research Network. Disponível em: <http://www.acrwebsite.org/search/view-conferenceproceedings.aspx?ld=6915>. Acesso em: 23/04/14.

Pesquisa Nacional de Endividamento e Inadimplência do Consumidor (PEIC) Disponível em: <www.cnc.org.br> . Acessado em: 17 de junho de 2014.

Souza, Marcos Aguerri Pimenta de (2013). O Uso do Crédito pelo Consumidor: Percepções Multifacetadas de um Fenômeno Intertemporal. Dissertação de Mestrado, Universidade de Brasília.

Tonetto, L. M. (2009). Racionalidade limitada e consumo: a configuração de objetivos na tomada de decisão do consumidor. Tese de Doutorado, Faculdade de Psicologia, Pontifícia Universidade Católica do Rio Grande do Sul, Porto Alegre, RS. 
Tonetto, Leandro Miletto, Brust, Priscila Goergen, \& Stein, Lilian Milnitsky. (2010). Quando a forma importa: o efeito de configuração de mensagens na tomada de decisão. Psicologia: Ciência e Profissão, 30(4), 766-779. Disponível em: <http://www.scielo.br/ scielo.php?script=sci_arttext\&pid=\$1414-98932010000400008\&Ing $=$ en\&tlng=pt. 10.1590/S1414-98932010000400008>. Acessado em 07/05/14.

Tversky, A., e Kahneman, D. (1981). The framing of decisions and the psychology of choice. Science, 211, 453-458.

Williams, P., Fitzsimons, G., e Block, L. (2004). When Consumers Do Not Recognize "Benign" Intention Questions as Persuasion Attempts. Journal of Consumer Research, 31(3), 540-550.

Artigo recebido em: 19/09/2015

Aprovado em: 17/12/2015 